Int. J. Dev. Biol. 49: 237-241 (2005)

doi: $10.1387 / \mathrm{ijdb} .041961 \mathrm{mt}$

\title{
Transfer of an avian genetic reflex epilepsy by embryonic brain graft: a tissue autonomous process?
}

\author{
MARIE-AIMÉE TEILLET ${ }^{*}, 1$, ROBERT NAQUET $^{2}$ and CESIRA BATINI ${ }^{3}$ \\ ${ }^{1}$ Laboratoire d'Embryologie Cellulaire et Moléculaire, CNRS UMR 7128, Nogent-sur-Marne, France, ${ }^{2}$ Institut de Neurobiologie Alfred \\ FESSARD, CNRS UPR 2197, Gif-sur-Yvette, France and ${ }^{3}$ Faculté de Médecine Pitié-Salpêtrière, Université Paris VI, \\ CNRS UMR 7091, Paris, France
}

\begin{abstract}
Electroencephalographic characteristics and clinical symptoms of an avian genetic reflex epilepsy have been transferred from Fayoumi epileptic (Fepi) chickens to non-epileptic chickens by embryonic homotopic grafts of brain neuroepithelium. Transplanted tissues belonging to the prosencephalic vesicle transferred epileptic electrical features while tissues from the mesencephalic vesicle were responsible for seizure motor manifestations of the disease. Thus each of these tissues can express their own specificity when grafted separately in a normal host, but they co-operate to produce the complete epileptic phenotype when grafted together.
\end{abstract}

KEY WORDS: Brain chimera, avian genetic reflex epilepsy, behavior transfer, EEG

\section{Introduction}

The quail-chick chimera system that Nicole Le Douarin developed at the end of the sixties (Le Douarin, 1969) became rapidly crucial for numerous aspects of experimental research in avian development. The principle of the method consists in exchanging in ovo precise embryonic regions between a quail donor and a chick host or conversely (homotopic grafts). Originally, this method took advantage of the specific structure of the quail interphase nucleus which shows a mass of heterochromatin associated with the nucleolus while in chick, like in most of other vertebrate species, heterochromatin is dispersed in the nucleoplasm. This particularity of the quail nucleus, which exists whatever the type of cell and the stage of development, was used as a natural cell marker to follow cell migrations during avian embryogenesis. More recently the use of a specific monoclonal antibody (QCPN, Hybridoma Bank) to recognize quail cells and the use of specific molecular markers renewed the method (see Le Douarin and Kalcheim, 1999).

One embryonic structure, the knowledge of which largely benefited from the quail-chick cell marking technique, is the neural crest, a cell population issued from the dorsal region of the neural tube and characterized by the capacity to migrate and to give rise to a variety of derivatives (see Le Douarin, 1982; Le Douarin and Kalcheim, 1999). In the experiments devoted to the neural crest study, a fragment of chick neural tube or neural folds was replaced in ovo by its quail counterpart before migration of crest cells i.e. from the 3-somite-stage (3-ss) to 25-ss, according to the rostrocaudal level considered (neural crest cells start to migrate progressively from rostral to caudal ends). The resulting chimeras were analyzed at sequential times during development up to crest cell definitive localization and differentiation.

Cell migrations within the central nervous system were analyzed in quail-chick chimeras during incubation (Balaban et al., 1988; Hallonet et al., 1990; Tan et al., 1991). Moreover, in order to observe the completion of brain and spinal cord organogenesis, chimeras were sacrificed at the end of incubation and even after hatching. Quail-chick chimeras operated at the brachial level of the spinal cord survived during one to three months before immunological rejection of the grafted tissues took place (Kinutani and Le Douarin, 1985; Kinutani et al., 1986). Brain chimeras obtained by exchanging definite regions of the encephalic neuroepithelium between quail and chick embryos at a developmental stage preceding vascularization of the encephalic vesicles (i.e. 10-12-ss) were also able to hatch. They could survive in good health for one or two weeks, during which they showed normal behavior (Balaban et al., 1988). Interestingly, quail-chick chimeras which were operated in particular regions of the future brain expressed, under specific stimulation (hormonal), some species specific characteristics of quail song, demonstrating that it is possible to transfer a genetic behavior from one animal to another by embryonic brain grafts (Balaban et al., 1988).

While genetic manipulations in birds are scarce, various natural mutations have been found in chicken populations (Crawford,

Abbreviations used in this paper:EEG, electroencephalographic; Fepi, Fayoumi epileptic chicken; Fhtz, Fayoumi epileptic heterozygote; ILS, intermittent light stimulation; ISS, intense sound stimulation; JA, JA57 normal chicken; ss, somite stage.

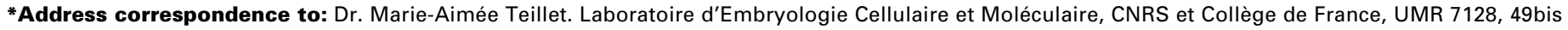
Avenue de la Belle Gabrielle, 94736 Nogent-sur-Marne Cedex, France. Fax: +33-1-4873-4377. e-mail: marie-aimee.teillet@college-de-France.fr
} 
1990). Among them, a mutation causing a reflex generalized epilepsy appeared spontaneously in a flock of Fayoumi chickens (Crawford, 1970). Fayoumi epileptic individuals were used to start an epileptic strain of chickens, called Fepi. Fepi mutants show, from hatching to adulthood, epileptic seizures induced by various stimuli and particularly by intermittent light stimulation (ILS). It was determined that Fepi is transmitted as an autosomal recessive mutation with high penetrance: all the Fepi homozygotes are epileptic, while the heterozygotes (Fhtz) do not show epileptic seizures (Crawford, 1970; Crichlow and Crawford, 1974).

We asked the question whether the Fepi phenotype was transferable to normal chickens through embryonic brain grafts. This experiment was made possible by the early expression of the disease after hatching. Moreover, a previous study on graft tolerance had shown that, in chicken to chicken combinations, chimeras survive for a long time without immune rejection (Ohki et al., 1987). Fepi and Fhtz eggs obtained from the original breeding allowed us to construct two separate flocks: a breeding stock of Fepi adults for the production of fertilized eggs destined to give homozygote Fepi embryos and another one of Fhtz individuals to preserve the mutation.

The experimental data reviewed in this article demonstrate that the origin of the different symptoms of the Fepi syndrome can be related to different neural structures spatially distributed within the chicken brain. Moreover, these results obtained by interspecific grafts allow us to discuss the autonomous development of particular neural tissue features in a foreign genetic environment.

\section{The Fepi mutation}

In a first part of our work (Guy et al., 1992), we confirmed clinical and electroencephalographic (EEG) data published by the group of Crawford (Crawford, 1970; Crichlow and Crawford, 1974). Like these authors, we observed that, in contrast to Fhtz and to normal
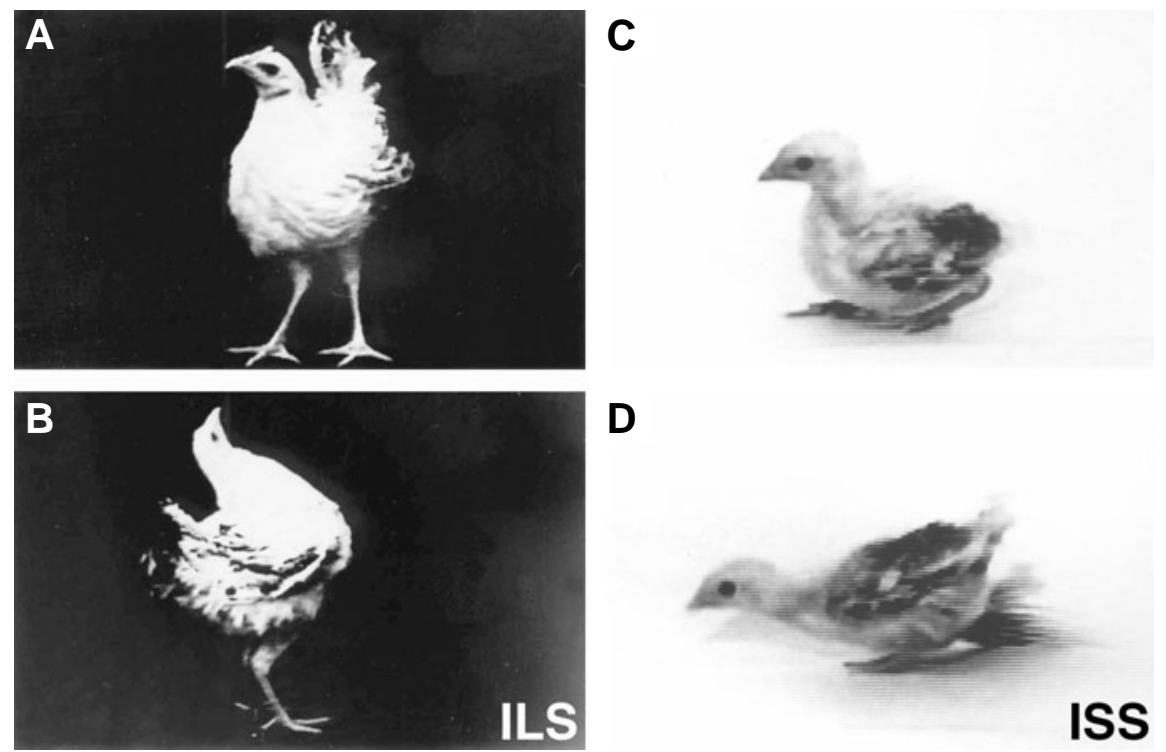

Fig. 1. Preconvulsive motor activities in Fepi animals under epileptogenic stimulations: intermitent light (ILS) and intense sound stimulation (ISS). Sequential images of ILSinduced neck myoclonus (A,B) and ISS-induced running fit (C,D) have been selected from video recordings. (Modified from Fadlallah et al., 1995). chickens of commercial source (JA57, ISA France, referred as JA), the Fepi resting EEG presents continuous paroxysmal abnormalities with spikes and spike-and-waves (see Fig. 4A1). We also observed that the epileptogenic stimulation induces the typical pattern of «generalized» epileptic motor seizures for which the Fepi was considered a model of the human «grand mal» (Crawford, 1990). Moreover, we found that during the ictal sequence, the EEG is characterized by the suppression of the resting paroxysmal activity which is therefore replaced by desynchronized EEG rhythms analogous to the «arousal reaction» (Moruzzi and Magoun, 1949) followed or not by flattening or depression of the activity (see Fig. $4 \mathrm{~A} 2$ ). This unusual EEG ictal activity is obtained consistently with the ILS stimulus at the frequency of 14 cycles/sec (Guy et al., 1992) as well as with the acoustic stimulus, intense sound stimulation (ISS) (Fadlallah etal., 1995). The clinical manifestations of the Fepi seizure have been divided into three phases: 1) «arousal» (ictal arousal), concomitant with ILS-induced neck myoclonus at the lightning frequency, or to the ISS-induced running fit (Fig. 1); 2) self-sustained generalized convulsions induced by either type of stimulation; 3) recovery period (Fadlallah et al., 1995). At the cellular level, paroxysmal bursting discharges, synchronous to the EEG spikes or spike-and-waves, were observed during the interictal phase in neurons of the «Wulst», a visual prosencephalic structure of the avian brain. During seizure, bursting activity was suppressed in these neurons, consistent with the desynchronization of the ictal EEG. In contrast, in the tectum, bursting neurons were observed only during the ictal period, synchronous with the light flashes (Guy et al., 1993).

\section{Construction of Fepi-JA brain chimeras}

We performed grafts of definite regions of the brain anlage from Fepi to JA embryos (Teillet et al., 1991), according to the method previously described for quail-chick brain chimeras (Balaban et al., 1988). Microsurgery was performed at 2 days of incubation corresponding to the stage of brain vesicle development (10-12ss). We constructed various types of brain chimeras depending on the brain vesicles considered (Fig. 2 A,C and Fig. 3). Our previous brain fate map constructed with the quail-chick cell marking technique (Balaban et al., 1988; Hallonet et al., 1990; Tan et al., 1991 and see Le Douarin et al., 1993) was used as a reference in Fepi-JA combinations for which there was no cell marker to distinguish host from donor tissues. However, the feather pigmentation, due to the neural crest cells included in the graft, showed in the region of the graft of the Fepi-JA chimeras, a distinct color which could serve as a topographical marker (Fig. 2 B,D) like in quail-chick chimeras (see Le Douarin et al., 1993). The rate of hatching was the same in Fepi-JA as in quail-JA chimeras: $10 \%$ as an average. In contrast to quail-chick chimeras, a complete tolerance of embryonic neural grafts between Fayoumi and JA chickens was observed, as expected (Ohki et al., 1987), so that Fepi-JA chimeras could survive to adulthood without immunological rejection. 
Among surviving chimeras, architecture brain malformation was revealed histologically post-mortem only in a few cases which were discarded. It should be noticed that neither chickens of the JA strain, nor any of the quail-chick chimeras described above, ever exhibited any epileptic manifestation, spontaneously or under any stimulation. Nevertheless, JA-JA chimeras were performed in addition as control (Teillet et al., 1991). They confirmed that embryonic brain grafting is not epileptogenic (see Fig. 3).

\section{Total transfer of the Fepi phenotype}

Grafts of the entire or almost the entire brain anlage (prosencephalon + mesencephalon + metencephalon) of Fepi embryos into JA embryos resulted in the transfer of the typical Fepi phenotype including EEG paroxysmal activity at rest, ictal EEG desynchronization, stimulus-specific preconvulsive symptoms (i.e. ILS-induced myoclonus and ISSinduced running fits), as well as the generalized convulsions followed by the recovery period (Fig. 3A $1,2)$. This means that the grafted neuroepithelium carries an intrinsic dysfunction which cannot be rescued by the environment of the normal nervous system during embryonic and postnatal development.

More restricted grafts were performed in order to assess the precise region or the minimum amount of brain graft necessary to obtain the same results: Transplants of the prosencephalic plus mesencephalic vesicles only (Fig. 2A,B; Fig. 3A3 and Fig. 4A) also transferred the whole epileptic phenotype of the donor. In contrast, transplants of the embryonic vesicles caudal to the mesencephalon (Fig. 3D) produced chimeras devoid of any light- or sound-induced epileptic manifestations.

Thus, the brain dysfunction leading to a total transfer of epileptic traits is not necessarily generalized to the whole brain.

\section{Dissociation of the Fepi characteristics}

Results described above prompted us to dissect further the neuroepithelium in order to know whether specific parts of the brain are responsible for the distinct epileptic manifestations that we described before.

Interestingly, chimeras having only the Fepi prosencephalon (Fig. 3B) exhibited, during resting time, the typical paroxysmal EEG with continuous spikes and spike-and-waves synchronous with the burst discharges of the underlying neurons (Fig. 4B). Moreover, this paroxysmal activity disappeared with ILS and ISS and was replaced by EEG desynchronization and arousal. However, these EEG manifestations were never accompanied by any abnormal motor behavior. In contrast, chimeras having a normal prosencephalon but the Fepi mesencephalon, with (Fig. 3C2) or without (Fig. 3C4) an Fepi metencephalon, showed a normal EEG at rest. Long-lasting neck myoclonus, although never followed by generalized convulsions, was induced by ILS, whereas running fits, sometimes followed by convulsions, were triggered by ISS
(Fig. 4C). These results demonstrate that in the absence of an epileptic prosencephalon, the mesencephalon must be considered as entirely responsible for ILS-induced myoclonus and ISS-induced running fits, the stimulus-locked symptoms. Moreover, it has to be considered as having a facilitating influence on the sound-induced generalized convulsions of the Fepi model of reflex epilepsy.

\section{Transferred avian genetic epilepsy is reminiscent of human genetic reflex epilepsies}

The above results show that, in Fepy mutation, defined regions of the brain anlage transfer distinct manifestations of the genetically transmitted disease: resting EEG and ictal EEG are linked to Fepi prosencephalon, whereas the paroxysmal motor symptoms are linked to Fepi mesencephalon, each one of these Fepi 


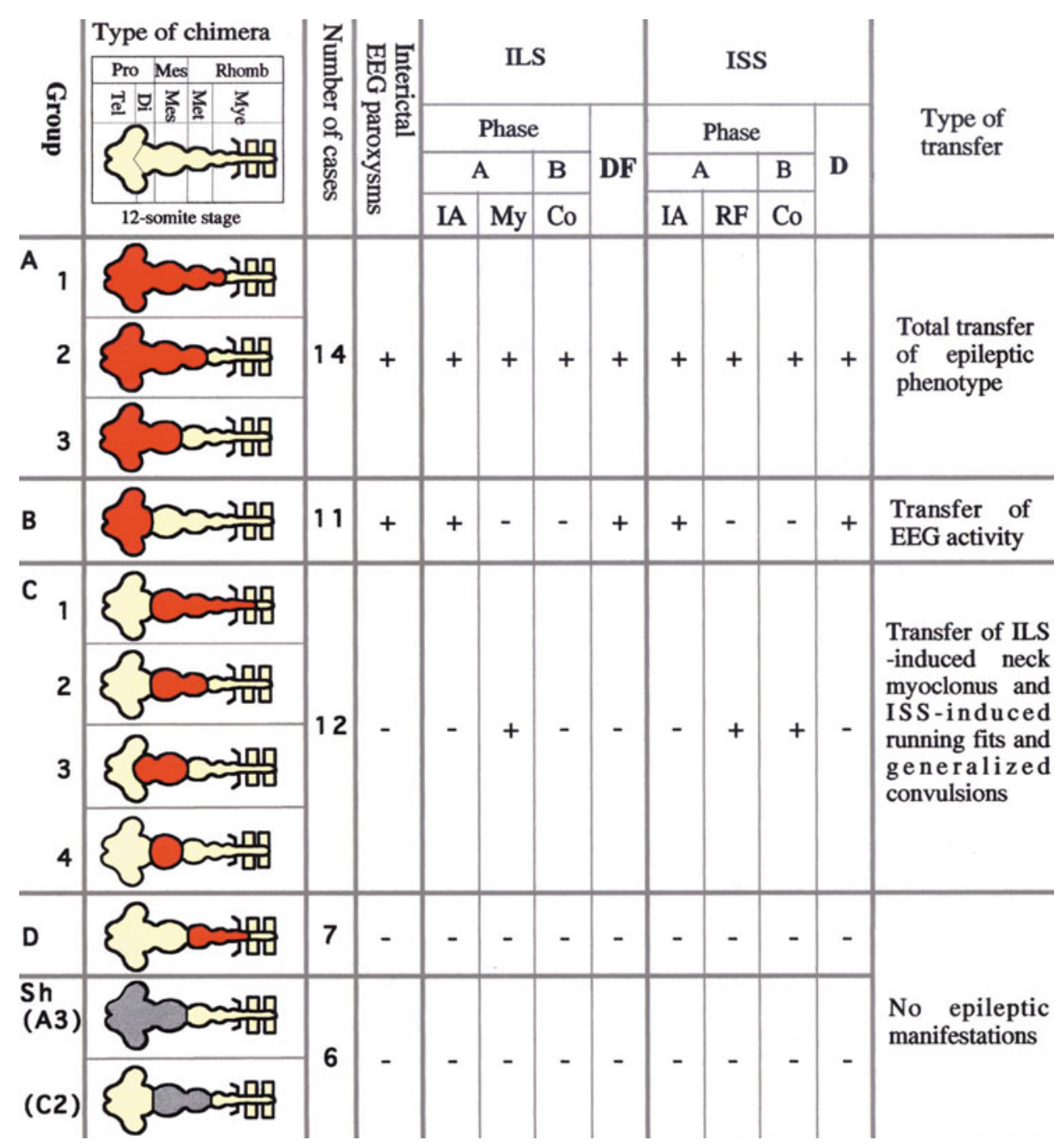

Fig. 3. Transfer of ILS- and ISS-induced epileptic electrographic (EEG) and clinical symptoms using homotopic grafts of various regions of the encephalic neuroepithelium. Experiments are made at the brain vesicle stage. Number of cases are indicated for the 4 groups of chimeras (A,B,C,D) and the two sham operations (Sh) in which wild type encephalic vesicles are grafted into wild type chick embryos according to the A3 and $C 2$ types of chimeras. Interictal EEG paroxysms are observed in all the chimeras having at least the Fepi prosencephalon (Pro) [groups (A, $\mathbf{B})$ ] and are never observed in chimeras having wild type Pro [groups (C,D)]. ILS- and ISS-induced ictal arousal (IA) are also restricted to groups (A) and (B). ILS-induced neck myoclonus (My) and ISS-induced running fits (RF), as well as ISS-induced convulsions (Co) appear in chimeras having at least the Fepi mesencephalon (Mes; groups (A,C)), while ILS-induced Co exist only in group (A) with at least Pro-Mes vesicles. Desynchronization of the EEG followed by flattening (DF) or not (D) are observed respectively under ILS and ISS in chimeras of groups $(\mathbf{A}, \mathbf{B})$ having at least the Fepi Pro. The type of transfer is recapitulated for each group of chimeras. Rhomb, rhombencephalon; ILS, intermittent light stimulation; ISS, intense sound stimulation (Modified from Batini et al., 1996). manifestations occurring independently of the other. The dissociation between EEG and clinical characteristics of the disease possibly owed to the existence of distinct neural networks probably carrying the same genetic defect.

These results allow to interpret certain human reflex syndromes like ILS-induced photomyologic response and «startle disease», also called hyperekplexia, induced by movement or surprise, for which it has been proposed that the reflex myoclonus response is of brainstem origin and does not involve the cortex (see Batini et al., 1996; Naquet and Batini, 2002).

\section{Discussion and Conclusion}

In the present article, we report how homotopic grafts of several regions of genetically epileptic brain anlage into normal chick embryos can be used to analyze different aspects of the avian genetic reflex epilepsy.

We demonstrated that the symptoms of the genetic disease could be transferred totally or partially depending of the grafted tissue. Presumably the totality of the Fepi neural tissue carries an identical genetic defect, but the resulting individual «pathological traits» appear diversified by the functional characteristics of individual mutated networks. We thus postulate that the conjunction of both of these conditions (genetic and functional) generates the epileptic symptoms. Moreover, the fate of the genetically mutated embryonic brain tissue is not influenced by the recipient developmental environment, meaning that the pathological mutation provides the tissue with an autonomous abnormal developmental process.

Recent researches on the transfer of genetically determined behavioral and physiological specificities, by encephalic neuroepithelium grafts in birds, support this interpretation. Thus, since the original work on the transfer of some aspects of the quail species-specific crowing to the chick, by the method of quail-chick chimeras (Balaban et al., 1988), this interspecific grafting method has pointed out the complexity of the species crowing behavior and the implication of definite regions of the brain in this process. It was discovered that two aspects of the same behavior, sound characteristics and head movements during crowing emission, are generated in two different regions of the brain, respectively the mesencephalon and the caudal part of the rhombencephalon (Balaban, 1997). Moreover, as for the Fepi genetic reflex epilepsy, these experiments have shown that the chick brain environment does not influence the expression of the quail specific genetic behavior. In other terms, quail crowing head movements can occur during chick crowing sound and conversely when the 
A

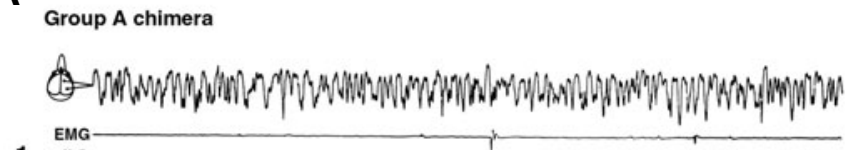

1 ILS
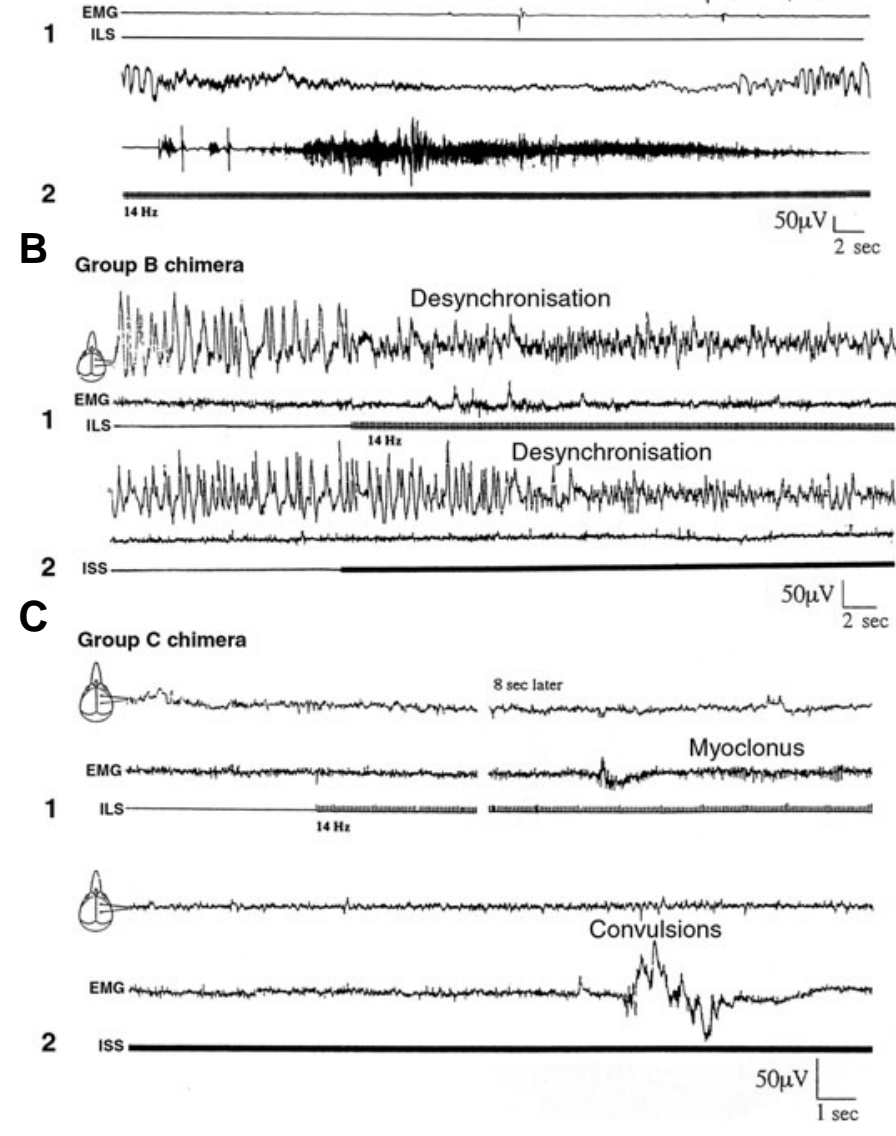

Fig. 4. Electroencephalographic (EEG, pictogram) and electromyographic (EMG) recordings in several types of chimeras. (A) Recordings of a chimera of group A (pro-mesencephalon, see Fig. 3A3): 1) EEG at rest presents typical Fepi interictal paroxysmal features; 2) ILS $(14 \mathrm{~Hz})$ starts EEG desynchronization and flattening with myoclonus. (B) Recordings of a chimera of group $B$ (prosencephalon alone, see Fig. 3B) shows desynchronization of the EEG paroxysms under ILS (1) or under ISS (2); (C) Recordings of a chimera of group C (mesencephalon alone, see Fig. 3C4) shows normal EEG at rest and under stimulations. ILS (1) and ISS (2) are followed respectively by unsustained myoclonus and convulsions. ILS, intermittent light stimulation; ISS, intense sound stimulation (Modified from Guy et al., 1992).

adequate regions of the brain are grafted separately. More recent experiments have shown that the inborn perceptual auditory predisposition, another very important aspect of the genetic behavior in young birds, could also be transferred by interspecific brain grafts (Long etal., 2001). Recognition of congeneric crowing is thus supported by intrinsic characteristics of specialized neurons developed in a foreign environment (see Balaban 2005, in this issue).

All these experiments realized in the avian embryo suggest that, following homotopic grafts, the chimeric brain develops as a new entity in which the genetic specificity of the grafted tissue is not affected by its new genetic environment.

\section{References}

BALABAN, E. (1997). Changes in multiple brain regions underlie species differences in a complex, congenital behavior. Proc. Natl. Acad. Sci. USA 94: 2001-2006.

BALABAN, E., TEILLET, M.-A. and LE DOUARIN, N. (1988). Application of the quailchick chimera system to the study of brain development and behavior. Science 241: 1339-1342.

BALABAN, E. (2005). Brain switching: studying evolutionary behavioral changes in the context of individual brain development Int. J. Dev. Biol. 49: 117-124. doi: 10.1387/ ijdb.041934eb

BATINI, C., TEILLET, M.-A., NAQUET, R. and LE DOUARIN, N.M. (1996). Brain chimeras in birds: application to the study of a genetic form of reflex epilepsy. Trends Neurosci.19: 246-252.

CRAWFORD, R.D. (1970). Epileptiform seizures in domestic fowl. J. Hered. 61: 185188.

CRAWFORD, R.D. (1990). In Poultry breeding and genetics (R.D. Crawford). Elsevier, 257-272.

CRICHLOW, E.C. and CRAWFORD, R.D. (1974). Epileptiform seizures in domestic fowl. II. Intermittent light stimulation and the electroencephalogram. Can. J. Physiol. Pharmacol. 52: 424-429.

FADLALLAH, N., GUY, N., TEILLET, M.-A., SCHULER, B., LE DOUARIN, N.M., NAQUET, R. and BATINI, C. (1995). Brain chimeras for the study of an avian model of genetic epilepsy: structures involved in sound and light-induced seizures. Brain Res. 675: 55-66.

GUY, N., TEILLET, M.-A., SCHULER, B., LE GAL LA SALLE, G., LE DOUARIN, N., NAQUET, R. and BATINI, C. (1992). Pattern of electroencephalographic activity during light induced seizures in genetic epileptic chicken and brain chimeras. Neurosci. Lett. 145: 55-58.

GUY, N.T., BATINI, C., NAQUET, R. and TEILLET, M.-A. (1993). Avian photogenic epilepsy and embryonic brain chimeras: neuronal activity of the adult prosencephalon and mesencephalon. Exp. Brain Res. 93: 196-204.

HALLONET, M.E., TEILLET, M.-A. and LE DOUARIN, N.M. (1990). A new approach to the development of the cerebellum provided by the quail-chick marker system. Development 108: 19-31.

KINUTANI, M., COLTEY, M. and LE DOUARIN, N.M. (1986). Postnatal development of a demyelinating disease in avian spinal cord chimeras. Cel/ 45: 307-314.

KINUTANI, M. and LE DOUARIN, N.M. (1985). Avian spinal cord chimeras. I. Hatching ability and posthatching survival in homo- and heterospecific chimeras. Dev. Biol. 111: 243-255

LE DOUARIN, M.L. (1969). Particularités du noyau interphasique chez la caille japonaise (Coturnix coturnix japonica). Utilisation de ces particularités comme «marqueur biologique» dans les recherches sur les interactions tissulaires et les migrations cellulaires au cours de l'ontogénèse. Bull. Biol. Fr. Belg. 103: 435-452.

LE DOUARIN, N.M., (1982). The neural crest. Cambridge University Press, Cambridge.

LE DOUARIN, N.M., (1993). Embryonic neural chimaeras in the study of brain development. Trends Neurosci. 16: 64-72.

LE DOUARIN, N.M. and KALCHEIM, C. (1999). The neural crest (second edition). Cambridge University Press, Cambridge.

LONG, K.D., KENNEDY, G. and BALABAN, E. (2001). Transferring an inborn auditory perceptual predisposition with interspecies brain transplants. Proc. Natt. Acad. SCi. USA 98: 5862-5867.

MORUZZI, G. and MAGOUN, H.W. (1949). Brain stem reticular formation and activation of the EEG. EEG Clin. Neurophysiol. 1: 455-474.

NAQUET, R. and BATINI, C. (2002). Genetic reflex epilepsy from chicken to man: relations between genetic reflex epilepsy and movement disorders. In Epilepsy and movement disorders (R. Guerrini, J. Aicardi, F. Andermann and M. Hallett). Cambridge University Press, Cambridge, 29-46.

OHKI, H., MARTIN, C., CORBEL, C., COLTEY, M. and LE DOUARIN, N.M. (1987). Tolerance induced by thymic epithelial grafts in birds. Science 237: 1032-1035.

TAN, K. and LE DOUARIN, N.M. (1991). Development of the nuclei and cell migration in the medulla oblongata. Application of the quail-chick chimera system. Anat. Embryol. 183: 321-343.

TEILLET, M.-A., NAQUET, R., LE GAL LA SALLE, G., MERAT, P., SCHULER, B. and LE DOUARIN, N.M. (1991). Transfer of genetic epilepsy by embryonic brain grafts in the chicken. Proc. Natl. Acad. Sci. USA 88: 6966-6970. 